\title{
APLICABILIDADE DA ESCALA DE AVALIAÇÃO FUNCIONAL EM EQUOTERAPIA-ESTUDO PILOTO II
}

APPLICABILITY OF THE FUNCTIONAL EVALUATION SCALE IN HIPPOTHERAPYPILOT STUDY II

SANTOS, Marcos Antonio dos ${ }^{1}$; SOUSA, Adriele Tatiane de Oliveira ${ }^{1}$; COSTA, Patrícia Teixeira $^{2}$; OLIVEIRA, Carolina de Camargo ${ }^{3}$; CASANOVA, Manuela Amaral ${ }^{3}$.

${ }^{1}$ Graduando do Curso de Fisioterapia-Universidade São Francisco; ${ }^{2}$ Diretora do CampusUniversidade São Francisco; ${ }^{3}$ Professora do Curso de Fisioterapia-Universidade São

Francisco.

marcossantos1994@hotmail.com

RESUMO. Introdução: A Equoterapia foi regulamentada pela Associação Nacional de Equoterapia em 1989, sendo método que utiliza o cavalo nas áreas da saúde, educação e equitação para o desenvolvimento biopsicossocial de pessoas com deficiência, em abordagem interdisciplinar. Objetivo: Analisar a aplicabilidade da Escala de Avaliação Funcional em Equoterapia (EAFE), com base em estudo anterior e nova abordagem observacional. Método: Trata-se de estudo de corte transversal com avaliação observacional única de crianças e adolescentes em tratamento Equoterapêutico e consideração única a respeito da EAFE, por meio de questionário respondido pelos profissionais. Após aprovação do Comitê de Ética (n.: 2.493.814) e assinatura de Termos de Consentimento e Assentimento, os profissionais da Alffa foram orientados e avaliaram os praticantes com a EAFE; posteriormente responderam questionário elaborado pelos pesquisadores deste estudo quanto a aplicabilidade da EAFE. Resultados: Participaram do estudo 42 praticantes, 33 do gênero masculino, entre 2 e 17 anos de idade, diagnósticos clínicos diversos, prevalecendo Autismo e Atraso do Desenvolvimento Neuropsicomotor. A maioria dos praticantes (48\%) foi pontuada com desempenho gravemente comprometido e nenhum com desempenho adequado, segundo a EAFE. Participaram da aplicação da escala 4 funcionários; todos responderam questionário, sendo que a maioria considerou a EAFE eficaz e aplicável; com poucas críticas e sugestões. Conclusão: A maioria dos praticantes teve desempenho motor gravemente comprometido com a EAFE, que foi considerada aplicável pela maioria dos profissionais participantes, com sugestões de melhorias. É necessário que este instrumento seja validado para fundamentar sua utilização em prática clínica e pesquisas futuras.

Palavras-chave: Terapia Assistida por Cavalos; Fisioterapia; Incapacidade Funcional.

ABSTRACT. Introduction: Hippotherapy was regulated by the National Association of Equine Therapy in 1989, being a method that uses the horse in the areas of health, education and riding for the biopsychosocial development of people with disabilities, in an interdisciplinary approach. Objective: To study the applicability of the Functional Evaluation Scale in Equine Therapy (EAFE), based on a previous study and a new observational approach. Method: This is a cross-sectional study with a single observational evaluation of children and adolescents undergoing equine treatment and a single consideration regarding EAFE, through a questionnaire answered by the professionals. After approval by the Ethics Committee ( $\mathrm{n}$.: 2,493,814) and signing of Terms of Consent and Assent, Alffa professionals were instructed and evaluated practitioners with EAFE; later answered a questionnaire 
prepared by the researchers of this study about the applicability of EAFE. Results: A total of 42 practitioners participated in the study, 33 males, between 2 and 17 years of age, with different clinical diagnoses, with Autism and Delayed Neuropsychomotor Development being the most prevalent. The majority of practitioners (48\%) were scored with severely compromised performance and none with adequate performance, according to EAFE. Four employees participated in the application of the scale; all answered a questionnaire, and the majority considered the EAFE to be effective and applicable; with few criticisms and suggestions. Conclusion: Most practitioners had motor performance severely compromised with EAFE, which was considered applicable by most of the participating professionals, with suggestions for improvements. It is necessary that this instrument be validated to justify its use in clinical practice and future research.

Keywords: Horse-assisted Therapy; Physiotherapy; Functional Incapacity.

\section{INTRODUÇÃO}

No Brasil, o termo Equoterapia foi determinado pela Associação Nacional de Equoterapia (ANDE- BRASIL) para caracterizar todas as práticas que utilizam o cavalo para atividade equestre com objetivos relacionados à reabilitação e educação de pessoas com necessidades especiais (ANDE-BRASIL, 2010). Em 1989, foi regulamentada pela ANDEBRASIL e foi definida como método terapêutico e educacional que utiliza o cavalo nas áreas de saúde, educação e equitação buscando desenvolvimento biopsicossocial de pessoas portadoras de necessidades especiais com uma abordagem interdisciplinar (ALVES, 2009).

De acordo com a ANDE - BRASIL (2010), a Equoterapia é indicada para indivíduos com doenças genéticas, neurológicas, ortopédicas, musculares, sequelas de trauma e cirurgias, doenças mentais, distúrbios psicológicos e comportamentais e distúrbios de aprendizagem e linguagem. Para gerar beneficio físico, psíquico, educacional e social.

Existem algumas contraindicações para a prática de Equoterapia que devem ser respeitadas, como quadro inflamatório e infeccioso; cifose grave e escoliose acima de 40 graus; luxação de quadril; extensão cruzada de membros inferiores e espasticidade sem mobilidade; epilepsia com crises convulsivas não controladas; obesidade; alergia ao pelo do cavalo; medo excessivo e problemas comportamentais do praticante que coloquem em risco sua segurança ou da equipe (LIPORONI; OLIVEIRA, 2005).

$\mathrm{Na}$ avaliação fisioterapêutica em Equoterapia, o uso de algumas escalas tem sido bastante usadas como a escala de equilíbrio de Berg, Gross Motor Function Mensure (GMFM), Peabody Motor Scale $2^{\circ}$ edição (PDMS), Denver Developmental Screening test - II (DDST), Desempenho Motor e Funcional de Crianças com Paralisia Cerebral, Escala de Avaliação do tono muscular, Inventário de Avaliação Pediátrica de Incapacidade (PEDI), Software para Avaliação Postural (SAPO) e Baropodometria (ALVES, 2009). Ou seja, são as mesmas usadas no atendimento clínico de Fisioterapia ou áreas afins e em pesquisas clínicas experimentais. Não foi encontrada uma escala específica para o atendimento em Equoterapia, mesmo sendo uma prática terapêutica tão difundida no Brasil e no mundo.

Partindo dessa constatação, foi elaborado um instrumento chamado Escala de Avaliação Funcional em Equoterapia (EAFE), específico para esta prática, como Trabalho de Conclusão de Curso (TCC) - "Reabilitação em Neurologia Infantil" - da Universidade Estadual de Campinas (UNICAMP) (MORASI; NATUCCI, 2009). Posteriormente, este instrumento foi aplicado pela primeira vez para testar a sua funcionalidade em outro TCC, 
desta vez do Curso de Fisioterapia da Universidade São Francisco (USF) como estudo piloto I (LONZA; BARNEZE, 2011), ambos sob orientação da Profa. Carolina Camargo de Oliveira, que também orientou a presente pesquisa.

O objetivo deste trabalho foi analisar a aplicabilidade da Escala de Avaliação Funcional em Equoterapia (EAFE), com base em estudo anterior e nova abordagem observacional.

\section{METODOLOGIA}

Trata-se de um estudo do tipo corte transversal com avaliação observacional única de crianças e adolescentes em tratamento Equoterapêutico, além de consideração única a respeito da EAFE, por meio de questionário respondido pelos funcionários. $\mathrm{O}$ estudo foi aprovado pelo Comitê de Ética em Pesquisa (Parecer: 2.493.814) e foi realizado no Centro de Equoterapia Lineu Marques Assis (CELMA), localizado em Bragança Paulista - SP. Os critérios de inclusão deste estudo foram crianças e adolescentes inseridos no atendimento de Equoterapia com idade até 19 anos e 11 meses, de acordo com a classificação de adolescência de Gallaheu (2005); e os profissionais técnicos, ou seja, da área de saúde e educação, que efetivamente trabalham no atendimento dos praticantes no CELMA.

Foram excluídos do estudo praticantes que não assinaram o Termo de Assentimento Livre e Esclarecido, quando maiores de 12 anos; cujos pais ou responsáveis legais não assinaram o Termo de Consentimento Livre e Esclarecido, quando menores de 18 anos; praticantes e profissionais que não assinaram o Termo de Consentimento Livre e Esclarecido (TCLE), próprio para maiores de 18 anos; praticantes não colaborativos ou que tiveram medo do cavalo. Foi utilizada como instrumento de pesquisa a Escala de Avaliação Funcional em Equoterapia (EAFE), elaborada por alunas da pós-graduação da Universidade de Campinas (UNICAMP) em 2009. A EAFE tem classificação baseada no escore final de cada praticante, sendo $76 \%$ a $100 \%$ - desempenho motor e habilidade funcional adequada durante a atividade equestre; $51 \%$ a $75 \%$ desempenho motor e habilidade funcional levemente comprometido durante a atividade equestre; $26 \%$ a $50 \%$ - desempenho motor e habilidade funcional moderadamente comprometido durante a atividade equestre; 0 a $25 \%$ - desempenho motor e habilidade gravemente comprometido durante a atividade equestre.

Os responsáveis legais pelos praticantes, os próprios praticantes em atendimento no CELMA e os profissionais do Centro, foram convidados e receberam esclarecimento sobre este trabalho de pesquisa, para autorização ou não, que incluiu avaliação nas atividades Equoterapêuticas dos praticantes, por meio da EAFE.

A partir desta etapa, os profissionais, receberam orientações sobre a aplicação da EAFE por 30 minutos; e no momento da aplicação da EAFE com os praticantes, os pesquisadores estiveram presentes para auxiliar os profissionais esclarecendo também dúvidas eventuais. A EAFE foi aplicada pelo profissional que estava mais habituado a atender o praticante. Após a aplicação da EAFE, os profissionais receberam um questionário, elaborado pelos pesquisadores deste estudo, perguntando sobre a aplicabilidade da EAFE na rotina profissional, bem como críticas e sugestões de melhorias. Os resultados foram submetidos à análise estatística simples e descritiva.

\section{RESULTADOS E DISCUSSÃO}

Aplicação da EAFE com os praticantes 
O projeto teve a coleta de dados finalizada com uma amostra de 42 praticantes, sendo 33 do gênero masculino (79\%) e 9 do feminino (21\%), de 2 aos 17 anos de idade (com Média $8,16 \% \pm 2,96 \%$ ), com diagnósticos diversos, envolvendo transtornos motores, cognitivos, comportamentais e do neurodesenvolvimento. Na tabela 1 encontra-se a casuística do presente estudo e, posteriormente, gráficos ilustram melhor, aspectos relevantes.

Tabela 1 - Dados de identificação dos praticantes e resultados da EAFE.

\begin{tabular}{|c|c|c|c|c|c|c|c|c|}
\hline Pets & Gênero & Idade & Diagnostico & $\begin{array}{l}\text { EAFE } \\
\text { part. 1 }\end{array}$ & $\begin{array}{l}\text { EAFE } \\
\text { part. } 2\end{array}$ & $\begin{array}{l}\text { EAFE } \\
\text { part.3 }\end{array}$ & $\begin{array}{l}\text { EAFE } \\
\text { Total }\end{array}$ & $\begin{array}{c}\text { Escore } \\
\text { Final }\end{array}$ \\
\hline 1 & $\mathrm{M}$ & $06 \mathrm{a}$ & Autismo & 4 & 3 & 0 & 7 & 10,14 \\
\hline 2 & M & $13 \mathrm{a}$ & Autismo & 18 & 6 & 0 & 26 & 37,68 \\
\hline 3 & M & $10 \mathrm{a}$ & Microcefalia & 9 & 4 & 0 & 13 & 18,84 \\
\hline 4 & $\mathrm{~F}$ & $05 \mathrm{a}$ & Autismo & 14 & 3 & 0 & 17 & 24,64 \\
\hline 5 & $\mathrm{~F}$ & $11 \mathrm{a}$ & Autismo & 16 & 7 & 0 & 23 & 33,33 \\
\hline 6 & $\mathrm{~F}$ & $09 \mathrm{a}$ & Síndrome de Down & 24 & 8 & 0 & 32 & 46,38 \\
\hline 7 & $\mathrm{M}$ & $07 \mathrm{a}$ & Hiperatividade & 25 & 8 & 0 & 33 & 47,83 \\
\hline 8 & M & $09 \mathrm{a}$ & Autismo & 16 & 6 & 0 & 22 & 31,88 \\
\hline 9 & M & $05 \mathrm{a}$ & $\begin{array}{l}\text { Paralisia Cerebral / } \\
\text { Hidrocefalia/ } \\
\text { ADNPM }\end{array}$ & 3 & 3 & 0 & 6 & 8,70 \\
\hline 10 & $\mathrm{~F}$ & $13 \mathrm{a}$ & Paralisia Cerebral & 7 & 3 & 0 & 10 & 14,49 \\
\hline 11 & M & $07 \mathrm{a}$ & Autismo & 19 & 5 & 0 & 24 & 34,78 \\
\hline 12 & M & $07 \mathrm{a}$ & Autismo & 4 & 3 & 0 & 7 & 10,14 \\
\hline 13 & M & $04 \mathrm{a}$ & Paralisia Cerebral & 5 & 4 & 0 & 9 & 13,04 \\
\hline 14 & M & $07 \mathrm{a}$ & Atraso DNPM & 26 & 10 & 0 & 36 & 52,17 \\
\hline 15 & M & $08 \mathrm{a}$ & Autismo & 10 & 2 & 0 & 12 & 17,39 \\
\hline 16 & M & $09 \mathrm{a}$ & Síndrome de Down & 25 & 8 & 0 & 33 & 47,83 \\
\hline 17 & M & $10 \mathrm{a}$ & $\begin{array}{l}\text { Paralisia Cerebral / } \\
\text { Síndrome West }\end{array}$ & 11 & 5 & 0 & 16 & 23,19 \\
\hline 18 & $\mathrm{~F}$ & $08 \mathrm{a}$ & Paralisia Cerebral & 3 & 4 & 0 & 7 & 10,14 \\
\hline 19 & $\mathrm{M}$ & $08 \mathrm{a}$ & Autismo & 10 & 4 & 0 & 14 & 20,29 \\
\hline 20 & M & $05 \mathrm{a}$ & $\begin{array}{l}\text { Transtornos Invasivos } \\
\text { do Desenvolvimento }\end{array}$ & 24 & 6 & 0 & 30 & 43,48 \\
\hline 21 & M & $07 \mathrm{a}$ & Autismo & 20 & 5 & 0 & 25 & 36,23 \\
\hline 22 & M & $05 \mathrm{a}$ & Autismo & 17 & 6 & 0 & 23 & 33,33 \\
\hline 23 & M & $05 \mathrm{a}$ & Autismo & 24 & 9 & 0 & 33 & 47,83 \\
\hline 24 & M & $08 \mathrm{a}$ & Autismo & 22 & 5 & 0 & 27 & 39,13 \\
\hline 25 & M & $07 \mathrm{a}$ & ADNPM & 21 & 6 & 0 & 27 & 39,13 \\
\hline 26 & M & $08 \mathrm{a}$ & Autismo & 23 & 10 & 0 & 33 & 47,83 \\
\hline 27 & M & $04 \mathrm{a}$ & Síndrome de Down & 3 & 3 & 0 & 6 & 8,70 \\
\hline 28 & M & $02 \mathrm{a}$ & Atraso DNPM & 10 & 3 & 0 & 13 & 18,84 \\
\hline 29 & M & $17 \mathrm{a}$ & Síndrome de Down & 25 & 5 & 0 & 30 & 43,48 \\
\hline 30 & M & $06 \mathrm{a}$ & Atraso DNPM & 13 & 5 & 0 & 18 & 26,09 \\
\hline 31 & M & $09 \mathrm{a}$ & Autismo & 9 & 2 & 0 & 11 & 15,94 \\
\hline 32 & M & $09 \mathrm{a}$ & Paralisia Cerebral & 5 & 3 & 0 & 8 & 11,59 \\
\hline 33 & $\mathrm{~F}$ & $07 \mathrm{a}$ & ADNPM & 10 & 6 & 0 & 16 & 23,19 \\
\hline 34 & M & $08 \mathrm{a}$ & Autismo & 26 & 6 & 0 & 32 & 46,38 \\
\hline 35 & M & $12 \mathrm{a}$ & Autismo & 20 & 7 & 0 & 27 & 39,13 \\
\hline 36 & $\mathrm{~F}$ & $13 \mathrm{a}$ & Paralisia Cerebral & 2 & 3 & 0 & 5 & 7,25 \\
\hline 37 & M & $07 \mathrm{a}$ & Autismo & 27 & 10 & 0 & 37 & 53,62 \\
\hline 38 & $\mathrm{~F}$ & $12 \mathrm{a}$ & Paralisia Cerebral & 3 & 5 & 0 & 8 & 11,59 \\
\hline 39 & M & $07 \mathrm{a}$ & Paralisia Cerebral & 12 & 4 & 0 & 16 & 23,19 \\
\hline 40 & M & $12 \mathrm{a}$ & Paralisia Cerebral & 12 & 5 & 0 & 17 & 24,64 \\
\hline 41 & M & $10 \mathrm{a}$ & Autismo & 18 & 8 & 0 & 26 & 37,68 \\
\hline 42 & M & $07 \mathrm{a}$ & Não Concluído & 16 & 5 & 0 & 21 & 30,43 \\
\hline
\end{tabular}

PCTS = pacientes; $\mathrm{M}=$ masculino; $\mathrm{F}=$ feminino; $\mathrm{a}=$ anos; EAFE = escala de avaliação funcional em equoterapia; $\mathrm{ADNPM}=$ atraso no desenvolvimento neuropsicomotor; DNPM: desenvolvimento neuropsicomotor. 
A EAFE é dividida em partes I, II e III, devendo a pontuação a ser somada para chegar ao valor total. Este valor obtido é dividido por 69 e em seguida multiplicado por $100 \%$ para obter o escore final. Quanto maior o escore final, melhor é o desempenho e habilidade funcional do praticante na atividade equestre.

O Gráfico 1 mostra a distribuição dos praticantes por grupos etários, revelando maioria de escolares $(53 \%, \mathrm{n}=20)$.

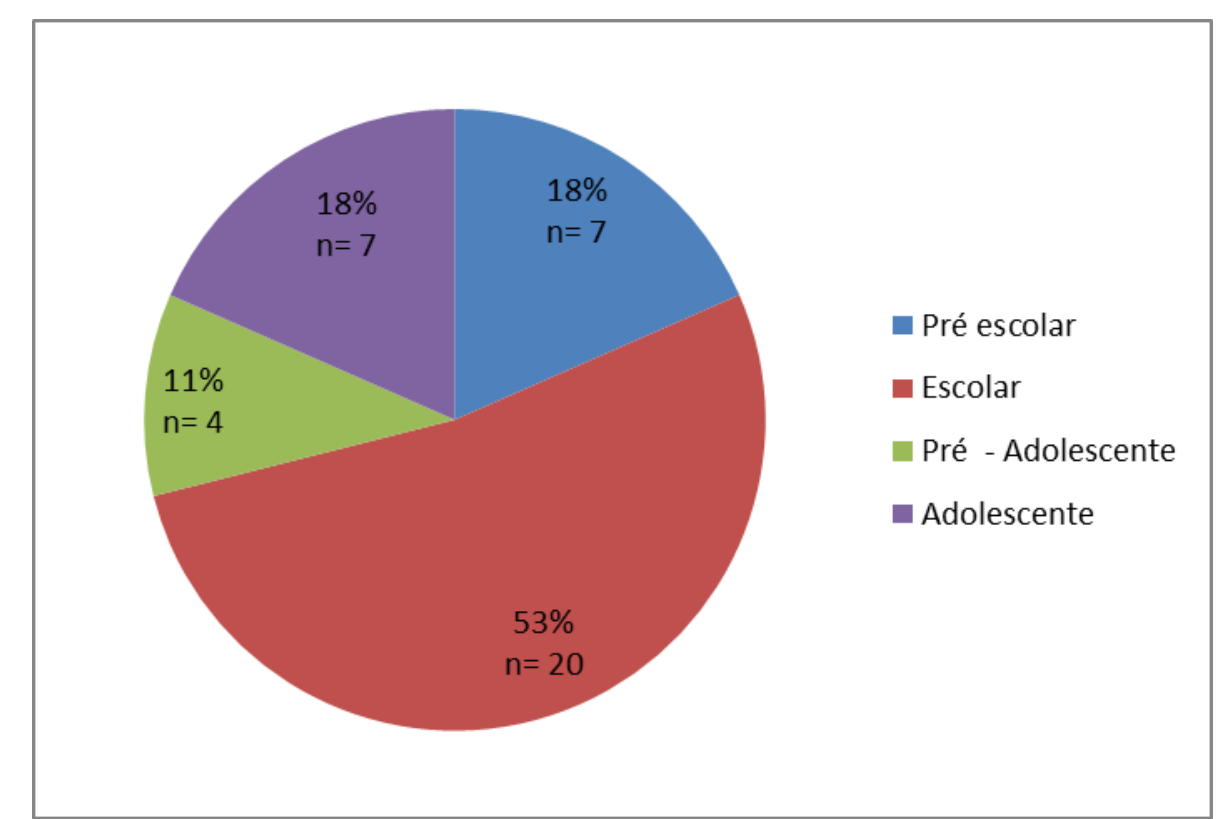

Gráfico 1 - Distribuição dos praticantes por grupos etários (Fonte: Próprio autor).

O Gráfico 2 revela a média e o desvio padrão das pontuações numéricas dos praticantes nas partes I, II e III da EAFE.

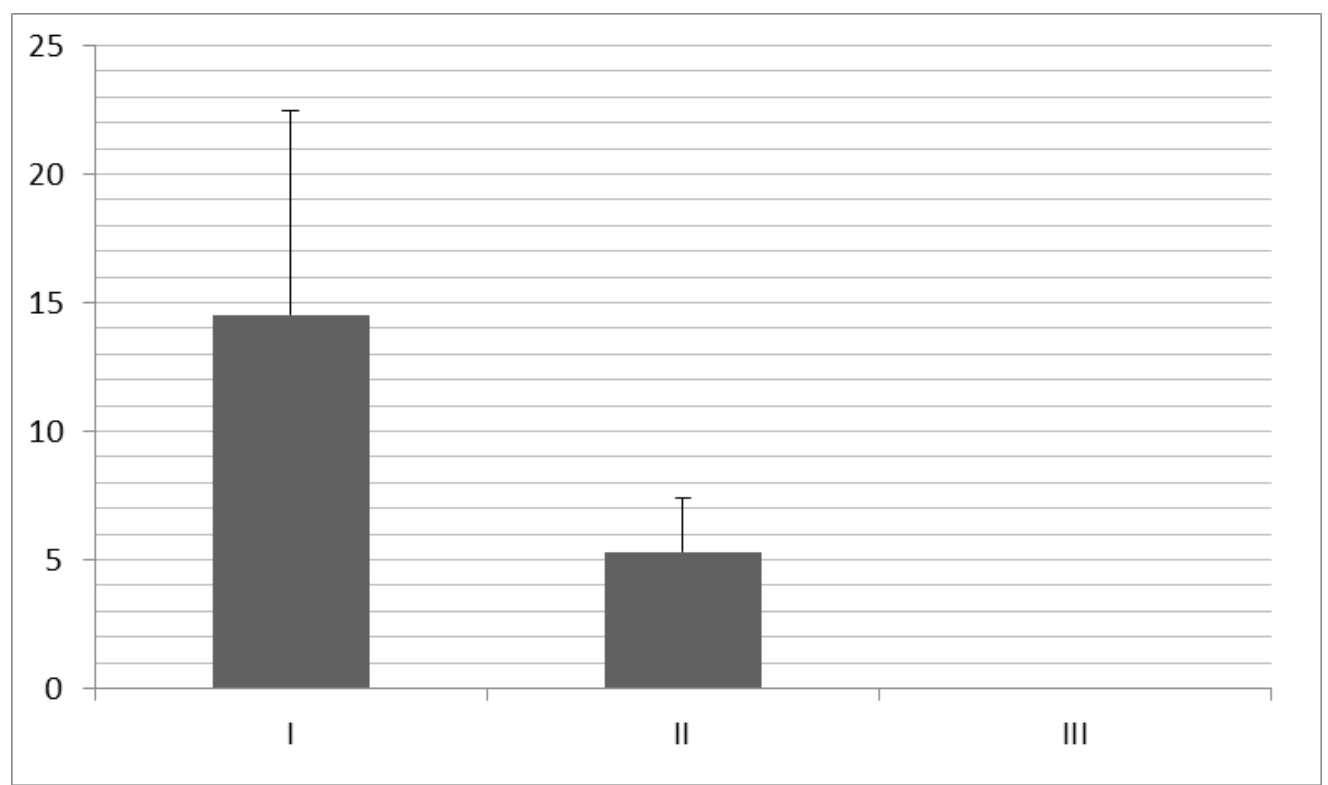

Gráfico 2 - Média e Desvio Padrão das pontuações dos praticantes nas partes I, II, III da EAFE (Fonte: Próprio autor). 
Na parte I a média de desempenho dos praticantes foi $14,5 \pm 7,9$; na parte II a média foi $5,3 \pm 2,1$; na parte III não foi obtido média e desvio padrão, já que ela não foi aplicada.

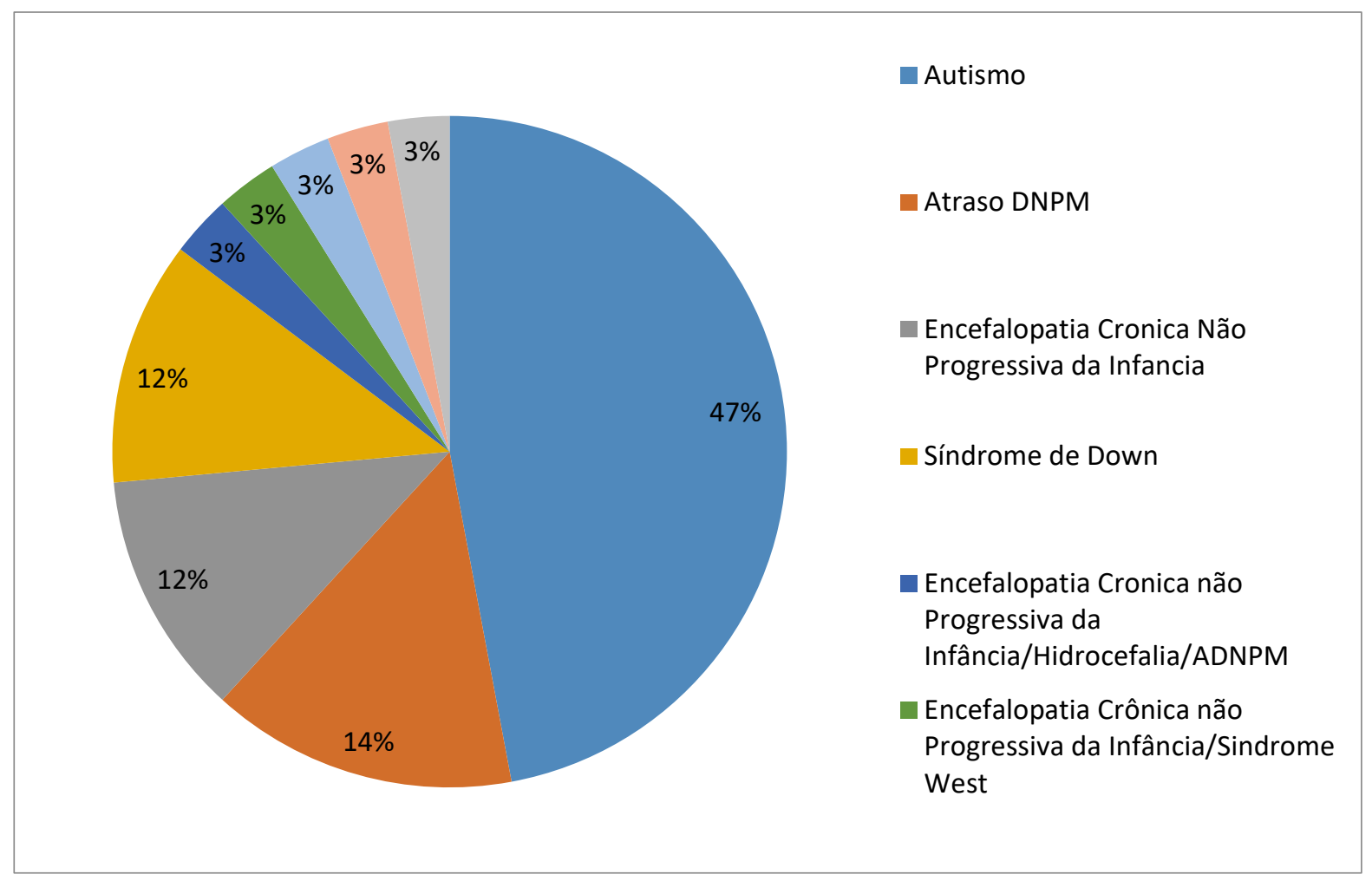

Gráfico 3 - Apresenta dos diferentes Diagnósticos dos praticantes participantes desta pesquisa (Fonte: Próprio autor).

Pode-se observar que os diagnósticos dos praticantes são diversos, porém há uma prevalência de Autismo (47\%, $\mathrm{n}=16)$, seguido de Atraso no Desenvolvimento Neuropsicomotor (DNPM) com 14\%. Os diagnósticos menos frequentes são Hiperatividades, Microcefalia e transtornos invasivos do desenvolvimento com apenas 3\%, conforme constava em prontuário durante coleta de dados.

O Gráfico 4 apresenta a porcentagem de quantos praticantes foram classificados em cada categoria da EAFE de acordo com seu escore, caracterizando desempenho motor adequado ou comprometido de forma leve, moderada ou grave. 


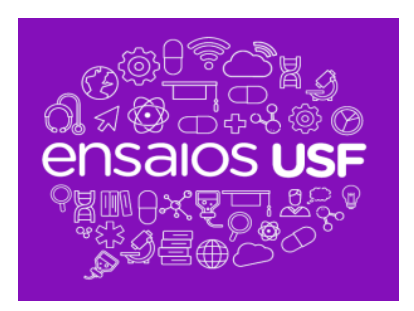

http://ensaios.usf.edu.br

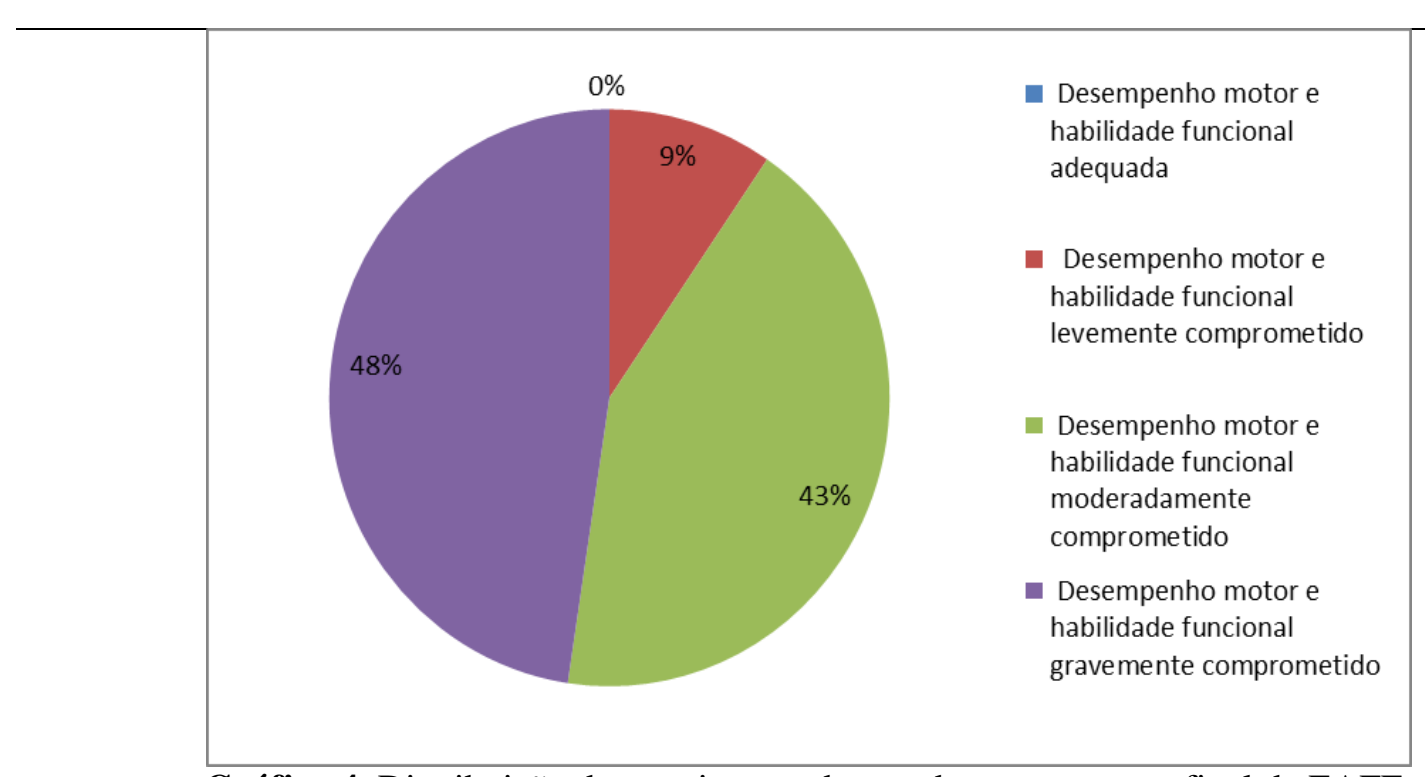

Gráfico 4. Distribuição dos praticantes de acordo com o escore final da EAFE.

Nenhum participante apresentou desempenho motor e habilidade funcional adequado (Escore final entre $76 \%$ e 100\%); a maioria apresentou desempenho gravemente comprometido ( $48 \%, \mathrm{n}=20$ ), seguido por desempenho moderadamente comprometido ( $43 \%$, $\mathrm{n}=18)$ e levemente comprometido $(9 \%, \mathrm{n}=4)$.

\section{Aplicação do questionário com os funcionários do CELMA}

Participaram da aplicação da EAFE 4 funcionários, sendo 3 fisioterapeutas e 1 psicólogo e todos devolveram o questionário que lhes foi entregue a respeito da aplicabilidade de tal instrumento. Foi detectado que dos 4 profissionais, 3 afirmaram que usariam EAFE no seu dia a dia, aplicando-a anualmente ou de 6 em 6 meses. Apenas um profissional relatou que não a usaria no seu dia a dia.

Os profissionais sugeriram mudanças para melhoria da escala, para uma futura validação. Algumas dessas sugestões foram, por exemplo, que a EAFE possa ser aplicada parcialmente, conforme o desempenho do praticante na atividade equestre. Por exemplo, a parte III da EAFE que avalia a habilidade de conduzir o cavalo, todos os praticantes foram pontuados com 0, pois avalia atividades que não são realizadas no CELMA devido ao importante comprometimento motor e cognitivo de todos ou da maioria dos praticantes.

Na parte I questão b2 (Figura 1), foram sugeridas mudanças em duas respostas; na pontuação "1" foi sugerida que se acrescentasse a "escovação com auxílio do terapeuta", pois muitas vezes o praticante realiza a escovação do cavalo, mas o terapeuta tem que auxiliá-lo. E a pontuação "0 - ele não realiza a atividade", deixa dúvida se o fator limitador são aspectos motores ou cognitivos. Muitas vezes o praticante tem capacidade motora para realizar a escovação, mas ele não faz essa atividade porque não compreende o que foi solicitado. 
Aspectos motores

b.2 - Escovação:

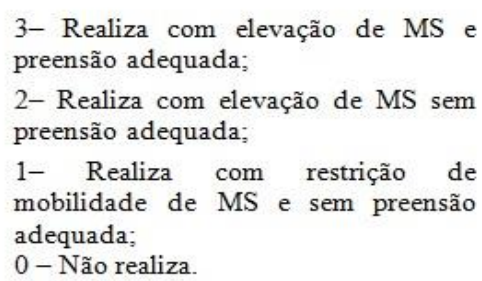

Figura 1- Questão b.2 da parte I da EAFE (Fonte: Próprio autor).

Na questão b3 (Figura 2), foi destacado que muitos praticantes não dão banho no cavalo devido à "patologia". Como por exemplo, praticantes com comprometimento motor e cognitivo grave, não conseguem executar essa atividade que, por isso, não é comumente usada nos atendimentos.

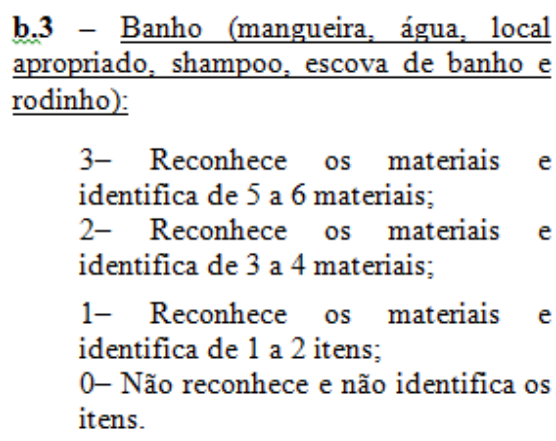

Figura 2- Questão b.3 da parte I da EAFE (Fonte: Próprio autor).

Na questão c1 (Figura 3) os profissionais do Centro de Equoterapia afirmaram que a alimentação não é feita por todos os praticantes devido à "patologia". E na questão c3 (Figura 4), destacaram que todos os praticantes que oferecem alimentos aos cavalos, acabam fazendo isso com auxílio do terapeuta, por medida de segurança.

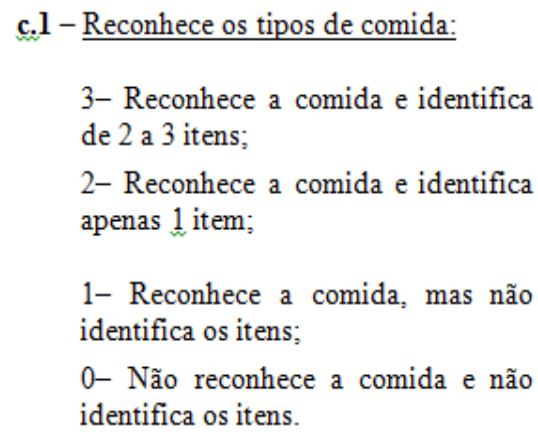

Figura 3- Questão c.1 da parte I da EAFE (Fonte: Próprio autor). 


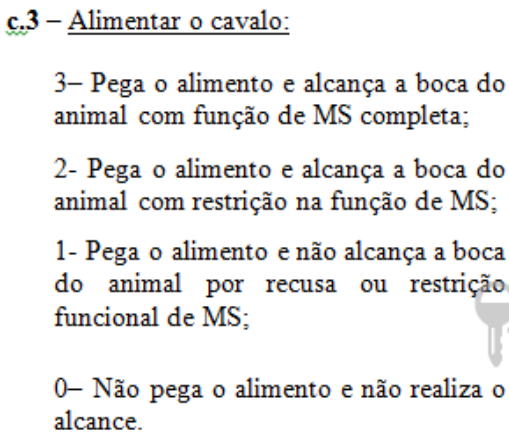

Figura 4- Questão c.2 da parte I da EAFE (Fonte: Próprio autor).

Este estudo teve uma amostra de 33 praticantes do gênero masculino e 9 do feminino totalizando 42 praticantes com diversos diagnósticos clínicos, envolvendo transtornos motores, cognitivos, comportamentais e do neurodesenvolvimento. A faixa etária variou 2 aos 17 anos de idade, com prevalência de escolares (6 a 12 anos) e diagnóstico clínico de Autismo, seguido pelo Atraso do Desenvolvimento Neuropsicomotor (ADNPM).

$\mathrm{O}$ número de praticantes que teve o desempenho motor e habilidade funcional gravemente comprometido foi de $48 \%$. A maioria dos praticantes teve pontuação baixa na parte II a escala, já que as atividades eram de maior complexidade, tendo desempenho gravemente comprometido. A parte III, mais complexa, não foi aplicada aos praticantes pelos profissionais, pois contempla atividades não realizadas nesse centro de Equoterapia. Apesar das mudanças sugeridas no estudo Piloto I e já efetuadas antes da utilização da EAFE no presente estudo, os profissionais do Centro de Equoterapia observaram que seria importante executar outras melhorias, como a possibilidade de aplicar a escala parcialmente. Assim os profissionais poderiam selecionar a parte da escala que mais se adequa a cada praticante, não sendo obrigatória a aplicação dela por inteiro. Seguindo essa sugestão, os praticantes teriam que ser divididos previamente, por suas habilidades nas tarefas relacionadas à prática equoterapêutica, possivelmente tendo como referência a divisão da própria Equoterapia (hipoterapia; reeducação equestre; pré-esportiva; e esportiva paraequestre), sendo necessário rever o método de pontuação para classificação final.

$\mathrm{Na}$ parte III da EAFE que avalia habilidades de conduzir o cavalo, não foram sugeridas mudanças na sua aplicação, já que o Centro de Equoterapia não realiza atividades paraequestre com os praticantes. Sendo assim, todos os praticantes foram pontuados com 0 em todas as atividades, baixando o escore final. Entretanto pode-se questionar se esse viés não faz parte da avaliação proposta pela EAFE, representando justamente essa falta de habilidade motora e/ou cognitiva dos praticantes para conduzir o cavalo. Mas, para essa constatação, é necessário que o serviço de Equoterapia tenha pelo menos tentado com que seus praticantes realizassem essas tarefas. Não é indicado pontuar positivamente ou negativamente qualquer atividade a ser avaliada, apenas pela inferência se o praticante deve ou não conseguir executála.

Com base na revisão bibliográfica, foi constado que nos estudos publicados, foram utilizadas escalas de uso clínico para avaliação de participantes em tratamento Equoterapêutico, específica para cada função avaliada. A maioria das pesquisas encontradas abordaram pacientes com diagnóstico de Encefalopatia Crônica Não Progressiva da Infância (ECNPI) usando as escalas Gross Motor Function Measure (GMFM) e a Gross Motor Function Classification System (GMFCS) para avaliar os praticantes de Equoterapia quanto a função motora grossa e, em todas elas, detectaram melhora no desempenho motor dos 
praticantes após o atendimento Equoterapêutico (PINA, LOUREIRO, 2006; LOUREIRO et al., 2010; NASCIMENTO et al., 2010; SILVA et al., 2015).

Os profissionais apontaram algumas mudanças que podem ser discutidas futuramente para validação da escala, na parte I na questão b2 eles sugeriram que acrescentasse uma resposta em que o praticante faz a escovação do cavalo com auxílio do terapeuta por segurança. Mas, se o praticante tem o controle de tronco e um cognitivo preservado, o profissional não precisa dar um auxílio ao praticante na atividade exercida, ele apenas pode ficar do lado do praticante caso ele não esteja acostumado com o cavalo. Ainda na mesma questão foi apontado que na última resposta o praticante pode não realizar a escovação por aspectos motores ou cognitivos. Mas nesta questão a interpretação pode ter sido feita de modo equivocado e, devido a isto, os profissionais não se atentaram que a atividade de escovação do cavalo já é avaliada por aspectos cognitivos e motores.

$\mathrm{Na}$ questão b3, eles alegaram que eles evitam fazer a atividade de dar banho no cavalo devido a aspectos motores e cognitivos comprometidos na maioria dos praticantes. Nas questões c1 e c3 eles apontaram algumas sugestões como a alimentação do cavalo, que deve ser feita com auxílio do terapeuta sempre que possível, para segurança do praticante. Mas em outros centros de Equoterapia, essa atividade é feita apenas com a supervisão próxima do profissional e não com auxílio físico, já sendo suficiente para a segurança do praticante.

Algumas sugestões dos profissionais são pertinentes, como a que indica a necessidade do profissional auxiliar fisicamente o praticante que tem habilidade motora comprometida, mesmo com o cognitivo preservado. Assim como, praticantes com função motora preservada e cognitiva afetada, também podem necessitar de auxílio até físico, pois podem não identificar o objeto ou sua função durante uma atividade relacionada aos cuidados do cavalo, por exemplo. Entretanto outras sugestões não são pertinentes, como a necessidade de auxílio mesmo quando o praticante tem comprometimento motor leve e cognitivo preservado. Entende-se que, nestes casos, a maioria das tarefas poderão ser executadas com autonomia e independência, embora muitas vezes, com supervisão próxima e segura. E essa opção de resposta já existe na EAFE.

Outros instrumentos de uso clínico foram encontrados em estudos sobre Equoterapia, incluindo o uso de instrumentos validados para a população adulta, mesmo em praticantes pediátricos. HOLANDA et al. (2013) utilizaram o Questionário de Avaliação Cognitiva em praticantes com Autismo e teve como resultado uma diminuição do nível cognitivo dos pacientes, mas é importante considerar vários fatores biopsicossociais associado ao Autismo para não interpretar de forma errada esses resultados. Já na pesquisa de BENDER; GUARANY (2016) a Escala de Medida Independência Funcional (MIF) e o Inventário de Avaliação Pediátrica de Incapacidade (PEDI) foram aplicados para avaliar o desempenho funcional das crianças e adolescentes com Autismo e teve como resultado ganho na área de autocuidado e mobilidade nas crianças após tratamento com Equoterapia.

Em outro trabalho com crianças diagnosticadas com ECNPI do tipo atetóide, a escala de Equilibrio de Berg (Berg Balance Scale) foi utilizada, revelando uma melhora significativa do desempenho motor no final da sessão de equoterapia, observando melhora qualitativa do alinhamento postural (CORRÊA et al.; 2012). No estudo de MORAES, A. G; COPETTI, F; ANGELO, V. R, et al. (2016), usaram a Escala de Equilíbrio de Berg e o PEDI para avaliar o efeito da Equoterapia no equilíbrio postural na posição sentado em crianças com ECNPI após realizar 24 sessões, e foi constado um ganho de desempenho motor nestas crianças que passaram pelas 24 sessões, melhorando equilíbrio postural na posição sentada e equilíbrio dinâmico. 
Portanto, justifica-se a elaboração e validação futura de um instrumento específico para a Equoterapia, como a EAFE, que possibilitaria avaliar de forma mais específica o desempenho dos praticantes nas tarefas relacionadas ao ambiente Equoterapêutico. Este instrumento vai permitir identificar dificuldades a serem trabalhadas, avaliando o praticante de forma mais ampla e não em funções específicas como realizado nos estudos encontrados. Desta forma, a EAFE tem o intuito de fornecer mais subsídios aos profissionais, auxiliando na elaboração de plano terapêutico e na constatação da evolução dos praticantes.

\section{CONCLUSÃO}

Conclui-se que a EAFE foi considerada aplicável pela maioria dos profissionais participantes, pois é de fácil entendimento e preenchimento, tendo sido considerada um bom complemento para outras avaliações. A disponibilidade de treinamento da EAFE facilitou a aplicação, pois foram esclarecidas as dúvidas dos profissionais. O desempenho da maioria dos praticantes nas atividades do ambiente Equoterapêutico, avaliado pela EAFE, foi considerado gravemente comprometido, não havendo qualquer praticante com desempenho considerado adequado.

A EAFE merece estudo mais aprofundado e futura validação para auxiliar a avaliação e elaboração de plano de tratamento fisioterapêutico mais específico, beneficiando principalmente os praticantes.

\section{REFERÊNCIAS}

ALVES. E. N. R. Prática em Equoterapia - Uma Abordagem Fisioterapêutica. Atheneu: São Paulo, 2009.

ANDE - BRASIL (Associação Nacional de Equoterapia), 2010. Disponível em: http://www.equoterapia.org.br/origem.php. Acesso em: 29 out, 2010.

AQUINO, F. J. M. Avaliação dos padrões de marcha e postura corporal dos praticantes de equoterapia com paralisia cerebral. Faculdade Latino Americana. Trabalho apresentado no $7^{\circ}$ Congresso Nacional de Iniciação Cientifica - CONIC - SEMESO, 2007 e aceito no Congresso Mundial de Educação Física - FIEP.

BENDER, D. D; GUARANY. N. R. Efeito da Equoterapia no Desempenho funcional de Crianças e Adolescentes com Autismo. Rev Ter Ocup Univ São Paulo-SP, v. 27, n. 3, p. 271-7, 2016.

CORRÊA, R. G; TONON. Érika; SUTER. T. M. C. A influência da equoterapia no Equilibrio de paciente com Paralisia Cerebral. Revista Hórus, v. 7, n.3, p. 1-8. 2012.

CALCATERRA, Valêria; VEGGIOTTI, Pierangelo; PALESTRINI, Clara. et al. PostOperative Benefits of Animal-Assisted Therapy in Pediatric Surgery: A Randomised Study. PLoS One, v. 10, n. 6, 2015.

GALLAHUE, D.L; OZMUN, J.C. Compreendendo o desenvolvimento motor: bebês, crianças, adolescentes e adultos. 3 ed. São Paulo: Phorte, 2005. 
HOLANDA, R. L; LIMA, F. S. P; LOBO,L. B. C, et al. Equoterapia e Cognição em pacientes Autistas: Estudo de Caso. Revista Expressão Católica. 2013 Jul./Dez; 2 (2): 83-96.

LIPORONI, G. F; OLIVEIRA, A. P. R. Equoterapia como Tratamento Alternativo para Pacientes com Sequelas Neurologicas. Revista Cientifica da Universidade de Franca, v.5, n.1 / 6, pp. 21-29, Jan 2003/ Dez 2005.

LONZA, A. L. R; BARNEZE. M. M. Aplicabilidade da Escala de Avaliação Funcional em Equoterapia- ESTUDO PILOTO I. Trabalho de Conclusão de Curso Realizado na Universidade São Francisco, Bragança Paulista - SP, 2011.

MORASI, T. M; NATUCCI, C. C. Proposta de um Instrumento de Avaliação Funcional Através de uma Revisão de Literatura. Trabalho Realizado na Faculdade de Ciências Médicas, UNICAMP; Campinas - SP, 2009.

MORAES, A. G; COPETTI, F; ANGELO, V. R. et al. The effects of hippotherapy on postural balance and functional ability in children with cerebral palsy. J. Phys Ther Sci., v. 28, n. 8, p. 2220-2226, 2016.

MENEGHETTI, C. H. Z; PORTO, C. H. S; IWABE, C, et al. Intervenção da Equoterapia no Equilíbrio Estático de Crianças com Síndrome de Down. Revista de Neurociência. Relato de Caso 2009.

NASCIMENTO, M. V. M; CARVALHO, I. S; ARAUJO, R. C. S. et al. O Valor da Equoterapia Voltada para Tratamento de Crianças com Paralisia Cerebral Quadriplegia. Brazilian Journal of Biomotricity, v. 4, $\mathrm{n}^{\circ} 1$, p 48-56, 2015.

PINA, L. V; LOUVEIRO, A. P. C. O GMFM e sua Aplicação na Avaliação Motora de Crianças com Paralisia Cerebral. Fisioterapia do Movimento. Curitiba, Abr/Jun, v.19-100, 2006.

QUEIROZ, C. O. V. Trabalho Tecnico Científico da ANDE - BRASIL. Visualização da Semelhança entre os Movimentos Tridimensionais do Andar do Cavalo com o Andar Humano. 2006, Bela Vista - MS.

SILVA, J. P; AGUIAR, O. X. Equoterapia em Crianças com Necessidades Especiais. Revista Cientifica Eletronica de Psicologia. Ano VI- Numero 11, Nov 2008.

SILVA, L. M; MONTEIRO, E. S; PAIVA, S. S. C. et al. Efeitos da Equoterapia da Função Motora Grossa de Pacientes com Encefalopatia Crônica Não Progressiva. Rev

Neurocienc.,v.23,n.1,p.16,2015.

Publicado em 17/12/2019 